\title{
Simultaneous implementation of antireflection and antitransmission through multipolar interference in plasmonic metasurfaces and applications in optical absorbers and broadband polarizers
}

https://doi.org/10.1515/nanoph-2020-0325

Received June 10, 2020; accepted August 11, 2020; published online August 27, 2020

\begin{abstract}
Metasurfaces have been widely used to control beam propagation e.g. transmission, reflection, and absorption on an interface through a thin layer of nanoantennas with the thickness smaller than the wavelength. However, previous study of metasurfaces typically focused on controlling only one form of these propagations. In this work, we propose and demonstrate a multipolar plasmonic metasurface that can simultaneously realize antireflection (AR) and antitransmission (AT) in the visible and nearinfrared regions. The AR and AT arise from destructive multipolar interferences in the backward and forward directions, respectively, i.e., through the generalized Kerker effect. By engineering the multipolar interference, we show that the AR and AT can happen at different or similar wavelength ranges, which can be used for low-absorption spectral filters due to off-resonance operation or inversely strong optical absorbers through near-resonance operation, respectively. We also present a simple twodimensional design of the multipolar metasurface that supports AT for one polarization and AR for another polarization over a broadband, which is applicable to broadband transmissive polarizers with efficiency over
\end{abstract}

Present address: Jihua Zhang, Nonlinear Physics Centre, Research School of Physics, The Australian National University, Canberra, ACT 2601, Australia.

*Corresponding author: Chunlei Guo, The Institute of Optics, University of Rochester, Rochester, New York 14627, USA, E-mail: guo@optics.rochester.edu. https://orcid.org/0000-00018525-6301

Jihua Zhang and Ran Wei, The Institute of Optics, University of Rochester, Rochester, New York 14627, USA. https://orcid.org/00000002-3506-6638 (J. Zhang)

Ә Open Access. ๑ 2020 jihua Zhang et al., published by De Gruyter. (c) BY International License.
$90 \%$ and the extinction ratio over $18 \mathrm{~dB}$. By tuning the dimension and thus the multipolar interference, the transmitted polarization and operation wavelength are both controllable.

Keywords: antireflection; antitransmission; generalized Kerker effect; multipolar metasurface; plasmonic metasurface.

\section{Introduction}

Metasurfaces consisting of two-dimensional arrangement of nanoantennas with subwavelength size, spacing, and thickness can manipulate the amplitude, phase, and polarization of light at will $[1,2]$. In terms of the amplitude manipulation, metasurfaces can control the transmission $(T)$, reflection $(R)$, and thus absorption $(A=1-T-R)$ of light through an interface. The amplitude control has been widely studied for realizing antireflection (AR) [3-9], antitransmission (AT) [10-15], and optical absorption (OA) [15-19] in various wavelength ranges. A fundamental and powerful approach to understand the underlying physics of this amplitude control is based on the multipolar expansion of the nanoantennas [20-28]. When a single nanoantenna or a nanoantenna array is irradiated by an external light field and multipole responses are excited in the nanoantennas, the reflection can be calculated by the interference between the radiation fields from multipoles in the backward direction, and the transmission can be calculated as the interference between the radiation fields from multipoles in the forward direction and the incident light. Therefore, the key idea to control the $T, R$, and $A$ lies in engineering the multipolar interference, i.e., using the generalized Kerker effect [7, 29-32]. Many functionalities have been reported based on the generalized Kerker effect. For example, AR [5-9, 33] and AT [10-13] can be realized 
through destructive multipolar interferences in the backward and forward directions, respectively. OAs have also been reported based on multipole engineering in lossy nanoantennas by suppressing all the forward transmission and backward reflection [28, 33-37]. However, the nanoantennas used in previous work are relatively thick in order to excite the high-order multipoles and thus complicated in fabrication. Furthermore, in most cases, each design can realize only one functionality, e.g., AR, AT or OA.

Recently, we reported a multipolar plasmonic metasurface for transmission wavefront control in the visible and near-infrared (IR) region with high efficiency by tuning the multipolar interference approaching the generalized Kerker condition [38, 39]. The proposed metasurface is easy to fabricate and the multipole response can be easily tuned through the dimension change. In this work, we engineer multipole response in the multipolar plasmonic metasurface and demonstrate a metasurface that can simultaneously support AR and AT in the visible and IR ranges. More interestingly, the AR and AT wavelengths can be tuned to be either different or near each other, which can be used for spectral filters with low absorption due to offresonance operation or inversely $\mathrm{OA}$ through nearresonance operation, respectively. In the first design, the transmission/reflection at the AR/AT wavelength $\sim 870 /$ $700 \mathrm{~nm}$ reaches $75.7 \% / 85.1 \%$ in simulation and $69.2 \% /$ $82.4 \%$ in experiment. In the second design, the absorption is up to $94.1 \%$ in simulation and $92.8 \%$ in experiment at the wavelength $\sim 695 \mathrm{~nm}$. Furthermore, a two-dimensional subwavelength grating design is presented here for transmissive polarizers by tuning the multipolar interference to realize AR for one polarization and AT for another polarization at the same wavelength range. More importantly, we can choose which polarization is the transmitted one and apply it to different wavelength ranges by tuning the multipolar interferences. We present transmissive polarizers operating at two wavelength ranges around 800 and $1060 \mathrm{~nm}$ with the transmission efficiency over 90\% and extinction ratio (ER) over $18 \mathrm{~dB}$ in broadband. This result indicates the possibility of applying plasmonic metasurfaces for certain optical functionalities with transmission efficiency over $90 \%$ in the visible and IR regions, where the efficiency of plasmonic metasurfaces is typically too low to support practical applications and thus all-dielectric metasurfaces are more commonly used [4042]. The record high transmission of plasmonic metasurfaces in this work is attributed to destructive multipolar interference in the backward direction and off-resonance operation of the multipole responses, which suppresses the reflection and reduces the absorption in the plasmonic nanoantennas, respectively. This multipolar plasmonic metasurface can find other applications in optical coatings, filters, and splitters.

\section{Theory and principle}

For a periodic array of nanoantennas with multipole responses, the multipole contributions of electric dipole (ED), magnetic dipole (MD), electric quadrupole (EQ), magnetic quadrupole (MQ), and electric octupole (EO) to the scattered fields are $[25,43]$

$$
\begin{aligned}
& E\left(p_{x, y}\right)=\frac{i k_{d}}{2 S \varepsilon_{0} \varepsilon_{d}} \cdot p_{x, y} \\
& E\left(m_{x, y}\right)=\frac{i k_{d}}{2 S \varepsilon_{0} \varepsilon_{d}} \cdot \frac{1}{v_{d}} m_{x, y} \\
& E\left(Q_{x z, y z}\right)=\frac{i k_{d}}{2 S \varepsilon_{0} \varepsilon_{d}} \cdot \frac{i k_{d}}{6} Q_{x z, y z} \\
& E\left(M_{x z, y z}\right)=\frac{i k_{d}}{2 S \varepsilon_{0} \varepsilon_{d}} \cdot \frac{i k_{d}}{2 v_{d}} M_{x, y} \\
& E\left(O_{x z z, y z z}\right)=\frac{i k_{d}}{2 S \varepsilon_{0} \varepsilon_{d}} \cdot \frac{k_{d}^{2}}{6} O_{x z z, y z z}
\end{aligned}
$$

where $p, m, Q, M$, and $O$ represent the complex ED, $\mathrm{MD}, \mathrm{EQ}$, $\mathrm{MQ}$, and EO moments, respectively. $S$ is the area of the unit nanoantenna. $\varepsilon_{d}$ and $k_{d}$ are the permittivity and wave number of the surrounding medium, respectively. $\varepsilon_{0}$ is the vacuum permittivity. When taking the substrate effect into consideration, the reflection and transmission coefficients can be calculated by $[7,25]$

$$
\begin{aligned}
& r_{x}=\frac{\left(1+r_{0} e^{i 2 k_{d} z_{0}}\right)\left[E\left(p_{x}\right)-E\left(M_{y z}\right)-E\left(O_{x z z}\right)\right]-\left(1-r_{0} e^{i 2 k_{d} z_{0}}\right)\left[E\left(m_{y}\right)-E\left(Q_{x z}\right)\right]}{E_{0}}+r_{0} e^{i 2 k_{d} z_{0}} \\
& t_{x}=\frac{E_{0}+E\left(p_{x}\right)+E\left(m_{y}\right)-E\left(Q_{x z}\right)-E\left(M_{y z}\right)-E\left(O_{x z z}\right)}{E_{0}} t_{0} e^{i k_{d} z_{0}}
\end{aligned}
$$


for $x$-polarization input along the positive $z$ direction, and

by:

$$
\begin{aligned}
& r_{y}=\frac{\left(1+r_{0} e^{i 2 k_{d} z_{0}}\right)\left[E\left(p_{y}\right)+E\left(M_{x z}\right)-E\left(O_{y z z}\right)\right]+\left(1-r_{0} e^{i 2 k_{d} z_{0}}\right)\left[E\left(m_{x}\right)+E\left(Q_{y z}\right)\right]}{E_{0}}+r_{0} e^{i 2 k_{d} z_{0}} \\
& t_{y}=\frac{E_{0}+E\left(p_{y}\right)-E\left(m_{x}\right)-E\left(Q_{y z}\right)+E\left(M_{x z}\right)-E\left(O_{y z z}\right)}{E_{0}} t_{0} e^{i k_{d} z_{0}}
\end{aligned}
$$

for $y$-polarization input along the positive $z$ direction. $E_{0}$ is the electric field of the normally incident plane wave at the point of the multipole expansion center. $z_{0}$ is the distance between the expansion center and the substrate interface. $r_{0}=\left(\sqrt{\varepsilon_{d}}-\sqrt{\varepsilon_{s}}\right) /\left(\sqrt{\varepsilon_{d}}+\sqrt{\varepsilon_{s}}\right)$ and $t_{0}=2 \sqrt{\varepsilon_{d}} /\left(\sqrt{\varepsilon_{d}}+\sqrt{\varepsilon_{s}}\right)$ are the Fresnel reflection and transmission coefficients of a normally incident plane wave at the surrounding-substrate interface, respectively. $\varepsilon_{s}$ is the permittivity of the substrate. Higher order of multipole moments such as magnetic octupole can also be included into the equations [24, 25]. The reflectance and transmittance of the periodic metasurface are

$$
R_{x, y}=\left|r_{x, y}\right|^{2}, \quad T_{x, y}=\sqrt{\varepsilon_{s} / \varepsilon_{d}}\left|t_{x, y}\right|^{2}
$$

Clearly, based on Eqs. (2) and (3), one can realize AT by utilizing only one multipole response such as the ED, or realize AR with two multipole responses such as ED and MD which is essentially the design principle of hightransmission Huygens' metasurfaces [44-49]. However, Huygens' metasurfaces typically require overlap of the ED and MD resonances, which results in a narrow operational bandwidth [9]. Furthermore, both singleresponse and dual-response Huygens' metasurfaces require operation near the resonances of the multipolar responses, which is normally accompanied by high absorption and thus limited transmission, particularly for plasmonic metasurfaces in the optical wavelength range $[44,46]$. On the other hand, through multipolar interference, it is possible to realize both AR and AT in multipolar metasurfaces even when all multipoles are off resonant, which can reduce the OA. Another advantage of the off-resonance operation lies in that the amplitude/phase variations of the multipolar responses over the spectrum are slower at wavelength ranges away from the resonance, which contributes to broader bandwidth. As we will show later, this is the main principle of the record high and broadband transmission for plasmonic metasurfaces demonstrated in this work. Lastly, we will show that OA can also be realized by overlapping the multipolar resonances in a similar wavelength range.

\section{Results and discussion}

Figure 1a shows the schematic of the proposed unit nanoantenna which is comprised a rectangle shaped gold nanoaperture and a gold nanorod separated by a perforated dielectric resist layer with an etched hole. The nanorod is situated in the etched hole. The nanoaperture, nanorod, and etched hole have the same dimension which is a rectangle with length of $l$ and width of $w$. The separation between the two gold layers is $t_{r} . t_{m}$ is the gold thickness. $P$ is the period of the array. The nanoantenna is located on a quartz substrate.

In the beginning, we show that this nanoantenna can simultaneously support AR and AT in the visible and IR region through the generalized Kerker effect. For this purpose, we propose a nanoantenna design with $P=320 \mathrm{~nm}$, $t_{m}=30 \mathrm{~nm}, t_{s}=180 \mathrm{~nm}, l=220 \mathrm{~nm}$, and $w=75 \mathrm{~nm}$. Figure $1 \mathrm{~b}$ shows the calculated transmission and reflection spectra of the nanoantenna array directly obtained from the power monitors in the simulation using Finite-Difference TimeDomain (FDTD) Lumerical. The input is normally incident from the nanoaperture side and linearly polarized along the width direction, that is the $y$ direction. In the simulation, the dielectric constants for the gold and quartz were directly obtained by using the Gold-Johnson and Christy database and the $\mathrm{SiO}_{2}$-Palik database in the software material's library, respectively. The resist is chosen as ZEP520 and the dielectric constants were obtained from ellipsometry data. We can see that AR is supported at $870 \mathrm{~nm}(T=75.7 \%, R=0)$ and AT is supported at $700 \mathrm{~nm}(T=0, R=85.1 \%)$. The AR and AT are realized through destructive multipolar interferences in the backward and forward directions, respectively. Figure 1c shows the multipole contributions to the transmission/reflection fields from ED, MD, EQ, MQ, and EO in this nanoantenna as 

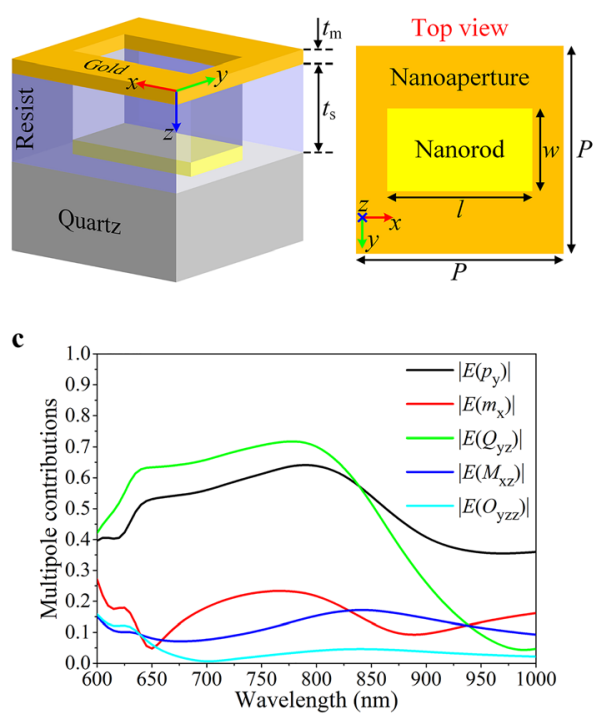

b

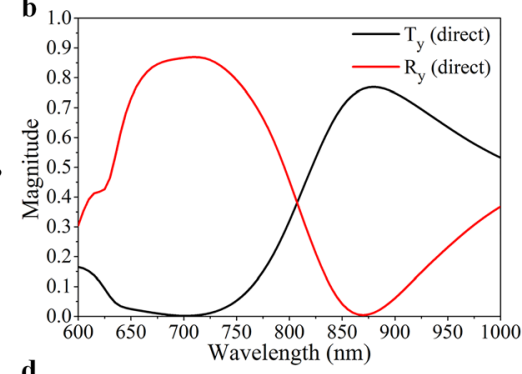

d

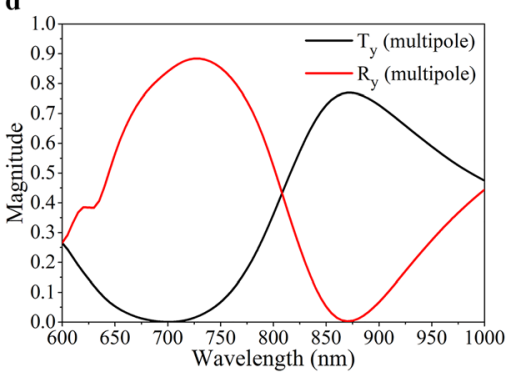

Figure 1: (a) Schematic of the unit nanoantenna of the multipolar plasmonic metasurface supporting simultaneous antireflection (AR) and antitransmission (AT) in the visible and near-infrared (IR) region. (b) Calculated transmission/reflection spectra through direct FDTD simulation for a nanoantenna array with $P=320 \mathrm{~nm}$, $t_{m}=30 \mathrm{~nm}, t_{s}=180 \mathrm{~nm}, l=220 \mathrm{~nm}$, and $w=75 \mathrm{~nm}$. (c, d) Calculated multipole contributions (c) to the transmission/reflection fields and their interference induced transmission/reflection spectra (d) for the designed nanoantenna array. The input source is from the nanoaperture side and linearly polarized along the $y$ direction. a function of the wavelength calculated using Eq. (1). We can see that the nanoantenna supports multipole response. Especially, the ED and EQ, and the MD and MQ have relatively similar magnitude in the wavelength range of interest, which facilitates the multipolar interference and the realization of AR and AT. Figure 1d shows the transmission and reflection spectra calculated by the multipolar interference using Eqs. (3) and (4). The multipole calculation also indicates AR at $\sim 870 \mathrm{~nm}$ and AT at $\sim 700 \mathrm{~nm}$, respectively, which shows agreement with the direct calculation in Figure $1 \mathrm{~b}$. The small discrepancy between the direct and multipole calculations might arise from the incompleteness of the multipole expansion methods we have used. It can be improved by including higher-order multipoles [26]. On the other hand, the substrate effect may be considered more precisely by considering the substrate-mediated inter-nanoantenna coupling and substrate-mediated selfcoupling [50]. More discussion on this is provided in the Supplementary material.

To show the multipolar interferences more clearly, we calculate the complex fields contributed from each multipole and direct transmission/reflection at the AR and AT wavelengths, and plot the vector superposition of these fields in Figure 2. Figure 2a, b shows the result for the transmission and reflection fields, respectively, at the AR wavelength of $870 \mathrm{~nm}$. Figure 2c, d shows the result for the reflection and transmission fields, respectively, at the AT wavelength of $700 \mathrm{~nm}$. In the calculation, we have made a global phase shift of all fields so that the direct transmission has a zero phase and the direct reflection has a $\pi$ phase. At the AR wavelength of $870 \mathrm{~nm}$, the multipolar interference induced transmission field (amplitude:
0.8769, phase: 0.2954 $)$ has a large amplitude (Figure 2a) while the reflection field (amplitude: 0.0544, phase: $-0.7018 \pi$ ) is small (Figure $2 b$ ), which indicates constructive and destructive interferences, respectively. In contrast, at the AT wavelength of $700 \mathrm{~nm}$, constructive and destructive multipolar interferences occur for the reflection (amplitude: 0.9167, phase: $0.1459 \pi$ ) (Figure 2c) and transmission (amplitude: 0.02, phase: $0.2691 \pi$ ) (Figure 2d), respectively. This result further confirms that the AR and AT of the proposed metasurface in Figure 1 are enabled by multipolar interferences, i.e., generalized Kerker effect. Here the overall phase of the transmission and reflection fields can be an arbitrary value in the range between 0 and $2 \pi$ by tuning the multipolar interference. Therefore, it is also possible to use this multipolar metasurface to realize full phase control for both the reflection and transmission fields with a unit amplitude [51].

We then experimentally fabricate the above metasurface design by using electron beam lithography and electron beam evaporation. The detailed fabrication procedure has been discussed elsewhere in a study by Zhang et al. [38]. The $T$ and $R$ of the sample in a broadband were measured by an optical setup schematically shown in Figure 3a. A collimated beam from a broadband lamp source (Thorlabs: SLS301) was focused onto the metasurface sample, which was mounted on a three-dimensional moveable and rotatable stage. Before the focusing lens, a broadband Glan-Thompson polarizer (Melles Griot: 03 PTH 112/C) was utilized to select the polarization. For the transmission measurement, the beam was collected by an objective $(10 \times, 0.25)$. For the reflection measurement, a small incident angle around $5^{\circ}$ was used in order to collect 
a

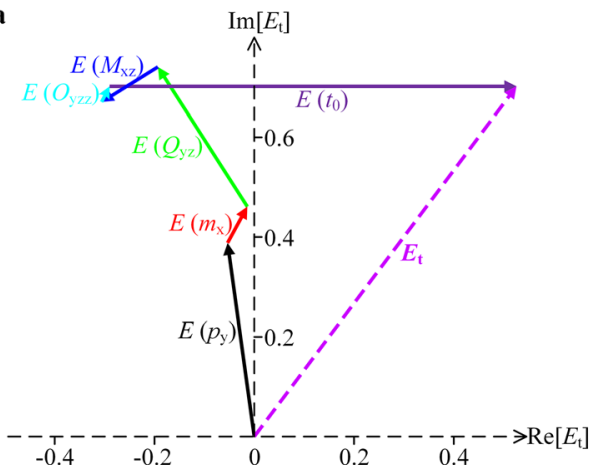

c

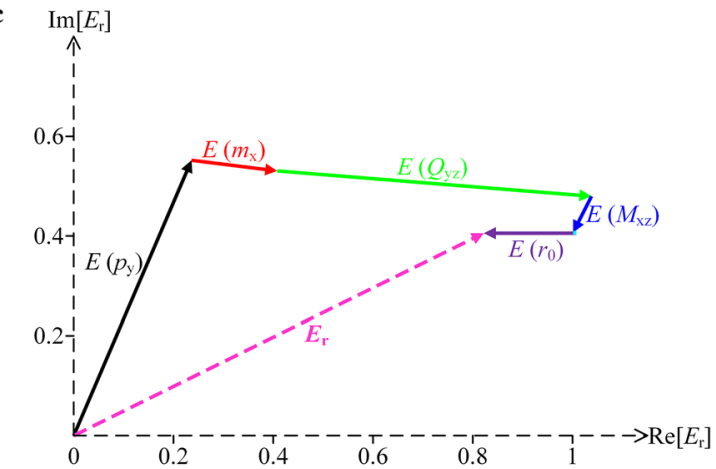

b

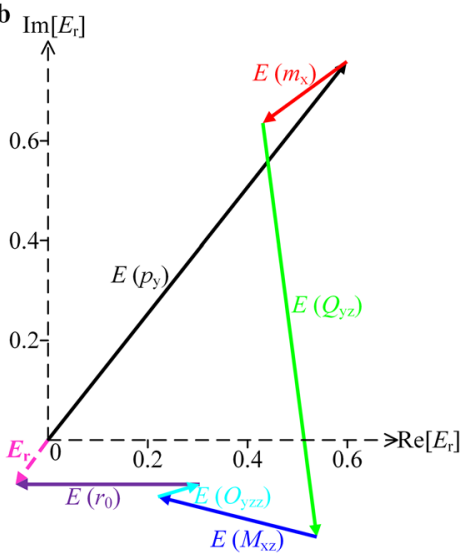

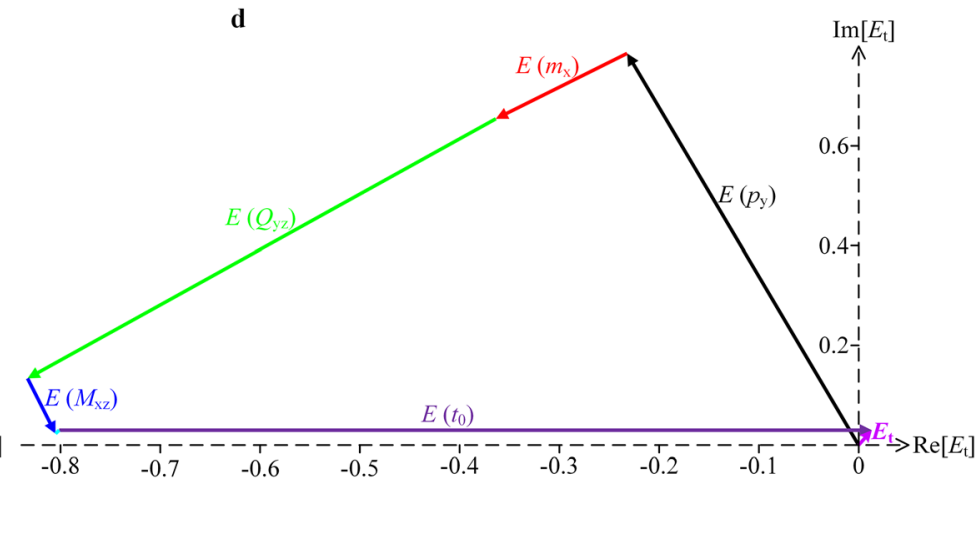

Figure 2: $(a, b)$ Vector superposition of the calculated fields contributed from each multipole and direct transmission/reflection in the transmission (a) and reflection (b) directions at $\lambda=870 \mathrm{~nm}$. (c, d) Vector superposition of the calculated fields contributed from each multipole and direct transmission/reflection in the reflection (c) and transmission (d) directions at $\lambda=700 \mathrm{~nm}$.
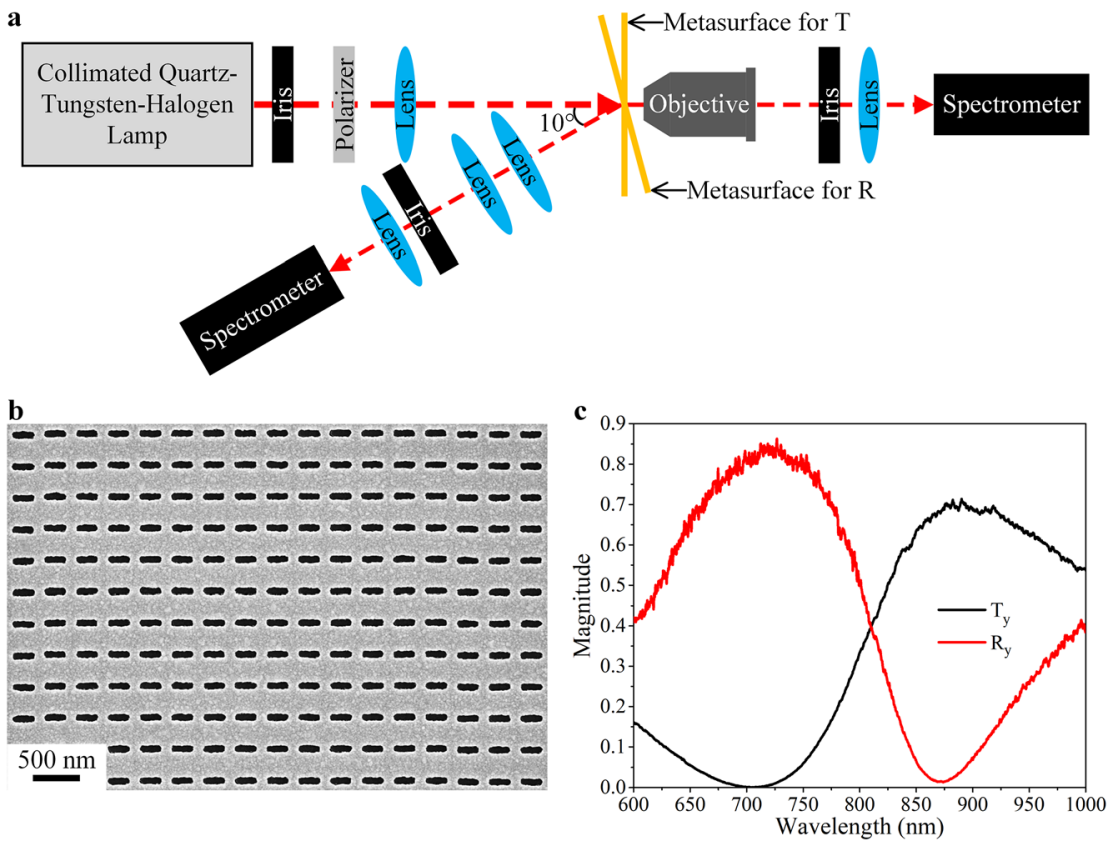

Figure 3: (a) Schematic of the optical setup for measuring the transmission/reflection spectra of the metasurface. (b, c) Scanning electron microscopy image (b) and measurement result (c) of the fabricated metasurface designed in Figure 1 for realizing antireflection (AR) and antitransmission (AT) simultaneously at different wavelengths in the visible and near-infrared (IR) region. 
all the reflected beams without blocking. Two lenses were utilized for the beam collection. The beam is finally focused into the fiber collector of a spectrometer (Photon Control: SPM001). An iris was used to select the part of the light through the metasurface area. Transmittance of the metasurface was normalized to the transmittance of the quartz substrate. Reflectance of the metasurface was normalized to the reflectance from a $100 \mathrm{~nm}$-thick gold thin film deposited on a quartz substrate. Figure 3b, c show the scanning electron microscopy image of the fabricated metasurface and the measurement result, respectively. In experiment, AT and AR are obtained at $\sim 705.7 \mathrm{~nm}(T=0$ and $R=82.4 \%)$ and at $\sim 873.9 \mathrm{~nm}(T=69.2 \%$ and $R=1.3 \%)$, respectively. This result is close to the simulation in Figure 1. At the AR wavelength, the minimum $R$ is not perfectly zero. This is due to the dimension difference in the fabrication and a small incident angle used in the measurement.

This above design indicates that the proposed metasurface can support AR and AT occurring at different wavelengths through multipolar interference. Next, we show that the AR and AT wavelengths are tunable by tuning the dimension. Especially, it is possible to tune them to an extreme case where the AR and AT wavelengths almost overlap with each other. In this case, the metasurface supports strong OA. The new design has $P=320 \mathrm{~nm}$, $t_{m}=30 \mathrm{~nm}, t_{s}=180 \mathrm{~nm}, l=122.5 \mathrm{~nm}$, and $w=90 \mathrm{~nm}$. Figure $4 \mathrm{a}, \mathrm{b}$ shows the calculated $T / R / A$ and multipole contributions of this design, respectively. We can see that the metasurface has $T$ lower than $10 \%$ in a wavelength range $645-708 \mathrm{~nm}$ with a minimum of $2.8 \%$ at $673 \mathrm{~nm}$. With respect to the $R$, it is lower than $10 \%$ in a similar wavelength range $687-703 \mathrm{~nm}$ with a minimum of zero at $695 \mathrm{~nm}$. The positions of the minimum wavelengths for $T$ and $R$ are close. As a result, strong absorption is supported in this wavelength range with $A>90 \%$ in $690-700 \mathrm{~nm}$ and a maximum value of $94.1 \%$ at $695 \mathrm{~nm}$. In Figure 4b, all the multipoles have similar magnitude in the wavelength range near $695 \mathrm{~nm}$, which causes AR and AT through multipolar interference. Meanwhile, all the multipoles support a local maximum in this wavelength range, which indicates resonances and thus strong absorption. Figure $4 \mathrm{c}$ shows the scanning electron microscopy image of the fabricated metasurfaces. The related transmission/reflection spectra of the metasurface are measured and shown in Figure 4d. In the experiment, $T$ and $R$ have minimum values of $3.8 \%$ at $711.2 \mathrm{~nm}$ and $2 \%$ at $692.4 \mathrm{~nm}$, respectively. As a result, the maximum $A$ is up to $92.8 \%$ at $692.8 \mathrm{~nm}$, near-perfect absorption is demonstrated. This experimental result is broader than the simulation in Figure 4a. This is due to the surface roughness of the deposited metal film, which causes lower quality factor of the multipole resonances and thus broader absorption. Note that the plasmonic metasurfaces demonstrated here are thinner than most of previous multipolar metasurfaces demonstrated for AR, AT, and $\mathrm{OA}$ in the similar wavelength range, while the performance is comparable $[9,11,35,36,47,52,53]$.

After utilizing the proposed multipolar metasurface to realize simultaneous AR and AT at one polarization,
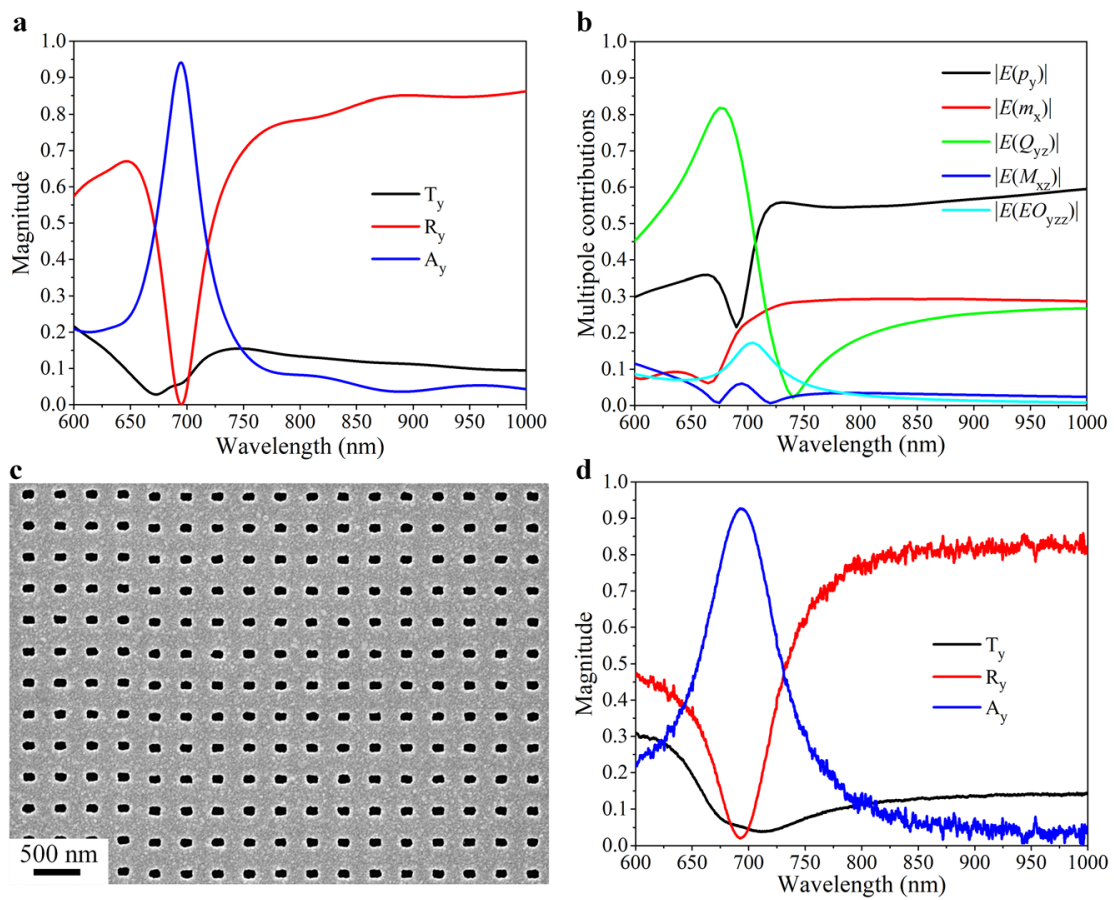

Figure 4: $(\mathrm{a}, \mathrm{b})$ Calculated transmission/ reflection/absorption spectra (a) and multipole contributions (b) for a nanoantenna array with $P=320 \mathrm{~nm}$, $t_{m}=30 \mathrm{~nm}, t_{s}=180 \mathrm{~nm}, l=122.5 \mathrm{~nm}$, and $w=90 \mathrm{~nm}$, which is designed with overlapped antireflection (AR) and antitransmission (AT) wavelengths and thus strong absorption at a visible wavelength. (c, d) Scanning electron microscopy image (c) and measurement result (d) of the fabricated metasurface. 
in the end we show that this metasurface can also be used to control the transmission and reflection of light at different polarizations. Specifically, we propose to use the metasurface for high-efficiency transmissive polarizers which have high transmission for one polarization and low transmission for another polarization. For this purpose, we let the length of the rectangle design to be infinite, i.e., a two-dimensional subwavelength grating design is used, as shown in Figure 5a. The two-dimensional structure is periodic in the $x$ direction and invariant in the $y$ direction. We first present two polarizers with optimized performance for the wavelength range around $800 \mathrm{~nm}$. The first polarizer design, which has $P=100 \mathrm{~nm}, w=42.4 \mathrm{~nm}$, $t_{s}=121.4 \mathrm{~nm}$ and $t_{m}=60 \mathrm{~nm}$, is a broadband polarizer that transmits the $x$-polarization and blocks the $y$-polarization. Its calculated transmission spectra of two polarizations and ER between two polarizations are shown in Figure $5 \mathrm{~b} . T_{y}$ is less than $1 \%$ in a broadband 665-1600 nm, while $T_{x}$ is over $72 \%$ in the same wavelength range with a peak value of $97 \%$ at $800 \mathrm{~nm}$. The ER which is defined by $10 \times \log _{10}\left(T_{x} / T_{y}\right)$ is more than 18.2 dB. We note that similar structure has been reported before for polarizer in the visible range [54]. However, here we explain the underlying physics of the selective transmission of $T_{X}$ through the generalized

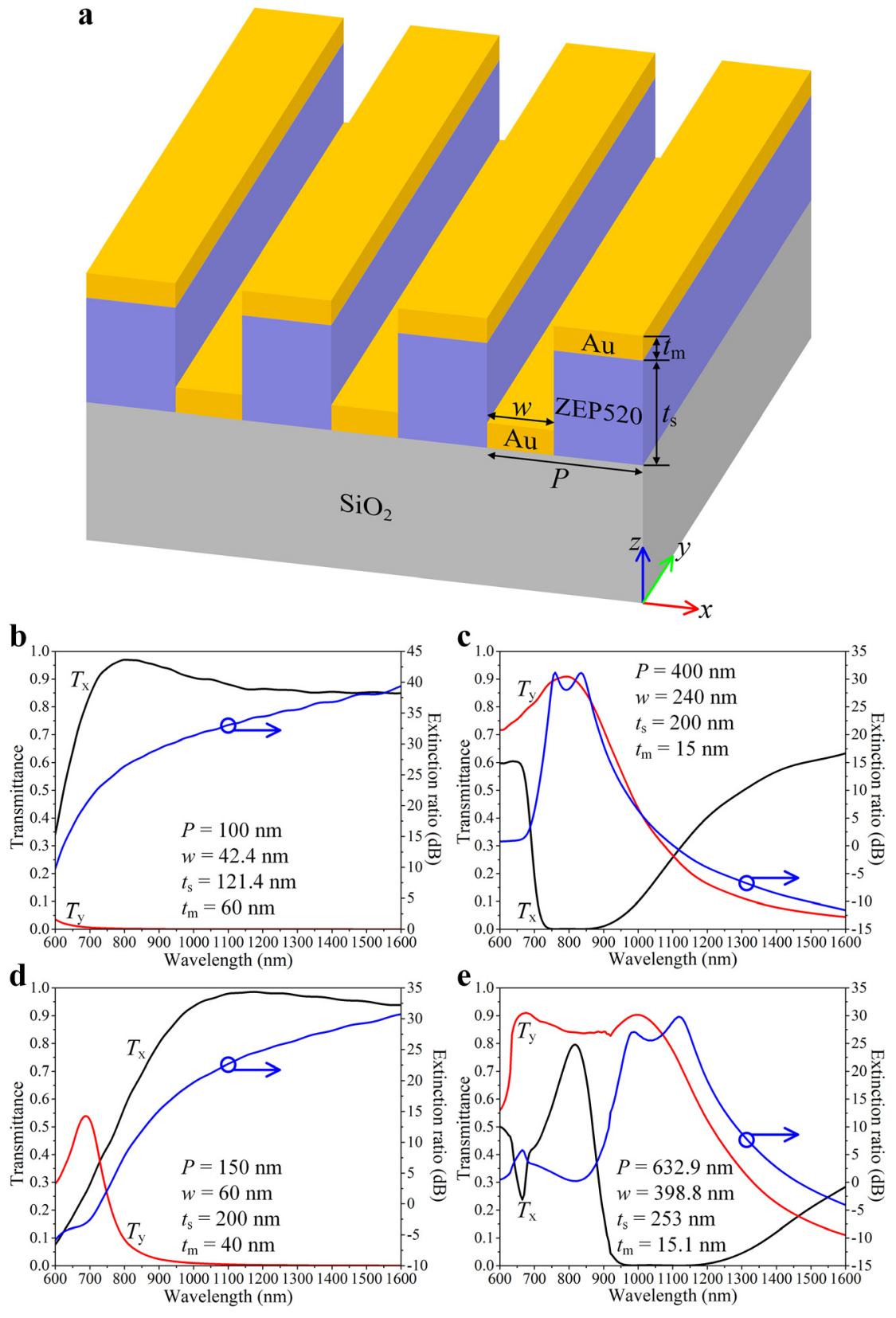

Figure 5: (a) Schematic of the twodimensional metasurface design for broadband polarizers. (b-c) Calculated $T_{x, y}$ and their extinction ratios of the polarizer designs optimized for transmission of $x$-polarization (b) and $y$-polarization (c) at a wavelength range $\sim 800 \mathrm{~nm}$. (d-e) Calculated $T_{x, y}$ and their extinction ratios of the polarizer designs for transmission of $x$-polarization (d) and $y$-polarization (e) at a wavelength range $\sim 1060 \mathrm{~nm}$. 
Kerker effect. Because of this, we are able to tune the multipolar interference of the metasurface for selective transmission of the $y$-polarization, which has never been reported before in a similar structure. The result of such a polarizer design is shown in Figure $5 \mathrm{c}$, which has $P=400 \mathrm{~nm}, w=240 \mathrm{~nm}, t_{s}=200 \mathrm{~nm}$ and $t_{m}=15 \mathrm{~nm}$. In a broadband $730-900 \mathrm{~nm}, T_{x}$ is less than $1 \%$, while $T_{y}$ is over $72 \%$ with a peak value of $91 \%$ at $800 \mathrm{~nm}$. The ER which is defined by $10 \times \log _{10}\left(T_{y} / T_{x}\right)$ exceeds $18 \mathrm{~dB}$. Furthermore, we can also design the polarizer optimized for other wavelength range of interest based on the multipole tuning. For example, Figure $5 \mathrm{~d}$, e show the polarizers optimized for a wavelength range $\sim 1060 \mathrm{~nm}$ for selective transmission of the $x$-polarization and $y$-polarization, respectively. The left design supports $T_{x}=98.4 \%$ and $\mathrm{ER}=24 \mathrm{~dB}$ at $1150 \mathrm{~nm}$. The right design supports $T_{y}=90.3 \%$ and ER $=26.6 \mathrm{~dB}$ at $1000 \mathrm{~nm}$. The simulations of the multipole contributions of these four polarizer designs are provided in the Supplementary material, which show the off-resonance of the multipolar responses at the operation wavelength of each design for the transmitted polarization. Compared to previous plasmonic metasurfaces enabled polarizers in the similar wavelength range, this $2 \mathrm{D}$ plasmonic metasurface is simpler, while the performance is comparable or even better [54-56].

These above results fully and clearly show the flexibility of the proposed multipolar metasurface in its structure, functionality, and operation wavelength for controlling the amplitude of the light at different polarizations with high efficiency. The fabrication of this metasurface is simpler than previous metasurfaces as there is no need for lift-off or etching after the lithography.

\section{Conclusion}

In conclusion, we have designed and demonstrated a multipolar plasmonic metasurface that can control the transmission, reflection, and absorption of light in the visible and IR region through multipolar interference. The metasurface is subwavelength in thickness. AR and AT have been realized simultaneously at different wavelengths or at close wavelengths, which can be used for spectral filter and OA, respectively. A simple two-dimensional design is further presented for realizing broadband polarizers at two different wavelength ranges near 800 and $1060 \mathrm{~nm}$. The polarizers support high transmission and ER with tunability on the transmitted polarization and operation wavelength. This metasurface will find applications in high-efficiency ultrathin optical coatings, filters, splitters, absorbers, and polarizers.
Acknowledgments: This work was supported by the US Army Research Office, DARPA, and Bill \& Melinda Gates Foundation. The fabrication described in this work was done in part at the Cornell NanoScale Facility, an NNCI member supported by NSF grant ECCS-1542081.

Author contribution: All the authors have accepted responsibility for the entire content of this submitted manuscript and approved submission.

Research funding: This work was supported by the US Army Research Office, DARPA, and Bill \& Melinda Gates Foundation. The fabrication described in this work was done in part at the Cornell NanoScale Facility, an NNCI member supported by NSF grant ECCS-1542081.

Conflict of interest statement: The authors declare no conflicts of interest regarding this article.

\section{References}

[1] A. V. Kildishev, A. Boltasseva, and V. M. Shalaev, "Planar photonics with metasurfaces,” Science, vol. 339, p. 1232009 , 2013.

[2] N. Yu and F. Capasso, "Flat optics with designer metasurfaces," Nat. Mater., vol. 13, pp. 139-150, 2014.

[3] M. Keshavarz Hedayati and M. Elbahri, “Antireflective coatings: conventional stacking layers and ultrathin plasmonic metasurfaces, a mini-review," Materials, vol. 9, p. 497, 2016.

[4] V. E. Babicheva, M. I. Petrov, K. V. Baryshnikova, et al., "Reflection compensation mediated by electric and magnetic resonances of all-dielectric metasurfaces [Invited]," J. Opt. Soc. Am. B, vol. 34, pp. D18-D28, 2017.

[5] R. Alaee, R. Filter, D. Lehr, et al., "A generalized Kerker condition for highly directive nanoantennas," Opt. Lett., vol. 40, pp. 2645-2648, 2015.

[6] S. Campione, L. I. Basilio, L. K. Warne, et al., "Tailoring dielectric resonator geometries for directional scattering and Huygens' metasurfaces," Opt. Express, vol. 23, pp. 2293-2307, 2015.

[7] A. Pors, S. K. H. Andersen, and S. I. Bozhevolnyi, "Unidirectional scattering by nanoparticles near substrates: generalized Kerker conditions,” Opt. Express, vol. 23, pp. 28808-28828, 2015.

[8] K. V. Baryshnikova, M. I. Petrov, V. E. Babicheva, et al., "Plasmonic and silicon spherical nanoparticle antireflective coatings," Sci. Rep., vol. 6, p. 22136, 2016.

[9] S. Kruk, B. Hopkins, Kravchenko, I. I., et al., “Invited Article: Broadband highly efficient dielectric metadevices for polarization control," APL Photonics, vol. 1, 2016, Art no. 030801.

[10] W. Liu, “Generalized magnetic mirrors,” Phys. Rev. Lett., vol. 119, p. 123902, 2017.

[11] P. Moitra, B. A. Slovick, W. Li, et al., "Large-scale all-dielectric metamaterial perfect reflectors," ACS Photonics, vol. 2, pp. 692-698, 2015.

[12] S. Liu, M. B. Sinclair, T. S. Mahony, et al., "Optical magnetic mirrors without metals,” Optica, vol. 1, pp. 250-256, 2014.

[13] W. Liu and A. E. Miroshnichenko, "Beam steering with dielectric metalattices,” ACS Photonics, vol. 5, pp. 1733-1741, 2018. 
[14] Z. Ma, S. M. Hanham, P. Albella, et al., "Terahertz all-dielectric magnetic mirror metasurfaces," ACS Photonics, vol. 3, pp. 1010-1018, 2016.

[15] T. Badloe, J. Mun, and J. Rho, "Metasurfaces-based absorption and reflection control: perfect absorbers and reflectors," $J$. Nanomater., vol. 2017, p. 2361042, 2017.

[16] G. M. Akselrod, J. N. Huang, T. B. Hoang, et al., "Large-area metasurface perfect absorbers from visible to near-infrared," Adv. Mater., vol. 27, pp. 8028-8034, 2015.

[17] R. Alaee, M. Albooyeh, and C. Rockstuhl, "Theory of metasurface based perfect absorbers,” J. Phys. Appl. Phys., vol. 50, p. 503002, 2017.

[18] A. K. Azad, W. J. M. Kort-Kamp, M. Sykora, et al., "Metasurface broadband solar absorber," Sci. Rep., vol. 6, 2016, https://doi. org/10.1038/srep20347.

[19] C.-H. Fann, J. Zhang, M. ElKabbash, et al., "Broadband infrared plasmonic metamaterial absorber with multipronged absorption mechanisms," Opt. Express, vol. 27, pp. 27917-27926, 2019.

[20] A. B. Evlyukhin, C. Reinhardt, and B. N. Chichkov, "Multipole light scattering by nonspherical nanoparticles in the discrete dipole approximation," Phys. Rev. B, vol. 84, p. 235429, 2011.

[21] A. B. Evlyukhin, C. Reinhardt, E. Evlyukhin, et al., "Multipole analysis of light scattering by arbitrary-shaped nanoparticles on a plane surface," J. Opt. Soc. Am. B, vol. 30, pp. 2589-2598, 2013.

[22] A. B. Evlyukhin, T. Fischer, C. Reinhardt, et al., "Optical theorem and multipole scattering of light by arbitrarily shaped nanoparticles," Phys. Rev. B, vol. 94, p. 205434, 2016.

[23] R. Alaee, C. Rockstuhl, and I. Fernandez-Corbaton, "An electromagnetic multipole expansion beyond the long-wavelength approximation," Optic Commun., vol. 407, pp. 17-21, 2018.

[24] R. Alaee, C. Rockstuhl, and I. Fernandez-Corbaton, "Exact multipolar decompositions with applications in nanophotonics," Adv. Opt. Mater., vol. 7, p. 1800783, 2019.

[25] P. D. Terekhov, V. E. Babicheva, K. V. Baryshnikova, et al., "Multipole analysis of dielectric metasurfaces composed of nonspherical nanoparticles and lattice invisibility effect," Phys. Rev. B, vol. 99, 2019, Art no. 045424.

[26] A. B. Evlyukhin and B. N. Chichkov, "Multipole decompositions for directional light scattering," Phys. Rev. B, vol. 100, p. 125415, 2019.

[27] E. A. Gurvitz, K. S. Ladutenko, P. A. Dergachev, et al., "The highorder toroidal moments and anapole states in all-dielectric photonics," Laser Photonics Rev., vol. 13, p. 1800266, 2019.

[28] R. Dezert, P. Richetti, and A. Baron, “Complete multipolar description of reflection and transmission across a metasurface for perfect absorption of light," Opt. Express, vol. 27, pp. 26317-26330, 2019.

[29] W. Liu and Y. S. Kivshar, "Generalized Kerker effects in nanophotonics and meta-optics [Invited]," Opt. Express, vol. 26, pp. 13085-13105, 2018.

[30] H. K. Shamkhi, K. V. Baryshnikova, A. Sayanskiy, et al., "Transverse scattering and generalized Kerker effects in alldielectric Mie-resonant metaoptics,” Phys. Rev. Lett., vol. 122, p. 193905, 2019.

[31] W. Liu and Y. S. Kivshar, "Multipolar interference effects in nanophotonics," Phil. Trans. Math. Phys. Eng. Sci., vol. 375, 2017, https://doi.org/10.1098/rsta.2016.0317.
[32] T. Liu, R. Xu, P. Yu, et al., "Multipole and multimode engineering in Mie resonance-based metastructures," Nanophotonics, vol. 9, p. 1115, 2020.

[33] H. K. Shamkhi, A. Sayanskiy, A. C. Valero, et al., "Transparency and perfect absorption of all-dielectric resonant metasurfaces governed by the transverse Kerker effect," Phys. Rev. Mater., vol. 3, 2019, Art no. 085201.

[34] Y. Ra'di, V. S. Asadchy, S. U. Kosulnikov, et al., "Full light absorption in single arrays of spherical nanoparticles," ACS Photonics, vol. 2, pp. 653-660, 2015.

[35] N. Odebo Länk, R. Verre, P. Johansson, et al., “Large-scale silicon nanophotonic metasurfaces with polarization independent near-perfect absorption," Nano Lett., vol. 17, pp. 3054-3060, 2017.

[36] C.-Y. Yang, J.-H. Yang, Z.-Y. Yang, et al., “Nonradiating silicon nanoantenna metasurfaces as narrowband absorbers," ACS Photonics, vol. 5, pp. 2596-2601, 2018.

[37] C. Liu, T. Wu, Y. Liu, et al., "Realization of perfect selective absorber based on multipole modes in all-dielectric moth-eye structure," Opt. Express, vol. 27, pp. 5703-5718, 2019.

[38] J. Zhang, M. ElKabbash, R. Wei, et al., "Plasmonic metasurfaces with $42.3 \%$ transmission efficiency in the visible," Light Sci. Appl., vol. 8, p. 53, 2019.

[39] C. Jin, J. Zhang, and C. Guo, "Metasurface integrated with double-helix point spread function and metalens for threedimensional imaging," Nanophotonics, vol. 8, p. 451, 2019.

[40] L. Zhang, S. Mei, K. Huang, et al., "Advances in full control of electromagnetic waves with metasurfaces," Adv. Opt. Mater., vol. 4, pp. 818-833, 2016.

[41] P. Genevet, F. Capasso, F. Aieta, et al., "Recent advances in planar optics: from plasmonic to dielectric metasurfaces," Optica, vol. 4, pp. 139-152, 2017.

[42] Q. He, S. Sun, S. Xiao, et al., "High-efficiency metasurfaces: principles, realizations, and applications," Adv. Opt. Mater., vol. 6, p. 1800415, 2018.

[43] V. E. Babicheva and A. B. Evlyukhin, "Analytical model of resonant electromagnetic dipole-quadrupole coupling in nanoparticle arrays," Phys. Rev. B, vol. 99, p. 195444, 2019.

[44] F. Monticone, N. M. Estakhri, and A. Alù, "Full control of nanoscale optical transmission with a composite metascreen," Phys. Rev. Lett., vol. 110, p. 203903, 2013.

[45] C. Pfeiffer and A. Grbic, "Metamaterial Huygens' surfaces: tailoring wave fronts with reflectionless sheets," Phys. Rev. Lett., vol. 110, p. 197401, 2013.

[46] C. Pfeiffer, N. K. Emani, A. M. Shaltout, et al., "Efficient light bending with isotropic metamaterial Huygens' surfaces," Nano Lett., vol. 14, pp. 2491-2497, 2014.

[47] M. Decker, I. Staude, M. Falkner, et al., "High-efficiency dielectric Huygens' surfaces,” Adv. Opt. Mater., vol. 3, pp. 813-820, 2015.

[48] A. Epstein and G. V. Eleftheriades, "Huygens' metasurfaces via the equivalence principle: design and applications," J. Opt. Soc. Am. A, vol. 33, pp. A31-A50, 2016.

[49] M. Chen, M. Kim, A. M. H. Wong, et al., "Huygens' metasurfaces from microwaves to optics: a review," Nanophotonics, vol. 7, p. 1207, 2018.

[50] K. M. Czajkowski, M. Bancerek, and T. J. Antosiewicz, "Multipole analysis of substrate-supported dielectric nanoresonator arrays with T-matrix method," https://arxiv.org/abs/2006.

$09137 v 12020$. 
[51] A. Rahimzadegan, D. Arslan, D. Dams, et al., "Beyond dipolar Huygens' metasurfaces for full-phase coverage and unity transmittance," Nanophotonics, vol. 9, p. 75, 2019.

[52] Y. Huang, H. Xu, Y. Lu, et al., "All-dielectric metasurface for achieving perfect reflection at visible wavelengths," J. Phys. Chem. C, vol. 122, pp. 2990-2996, 2018.

[53] J. Tian, H. Luo, Q. Li, et al., "Near-infrared super-absorbing alldielectric metasurface based on single-layer germanium nanostructures," Laser Photonics Rev., vol. 12, p. 1800076, 2018.

[54] Y. Ekinci, H. H. Solak, C. David, et al., "Bilayer Al wire-grids as broadband and high-performance polarizers," Opt. Express, vol. 14, pp. 2323-2334, 2006.
[55] H. Kurosawa, B. Choi, Y. Sugimoto, et al., "High-performance metasurface polarizers with extinction ratios exceeding 12000," Opt. Express, vol. 25, pp. 4446-4455, 2017.

[56] Y. Zhao, M. A. Belkin, and A. Alù, "Twisted optical metamaterials for planarized ultrathin broadband circular polarizers," Nat. Commun., vol. 3, p. 870, 2012.

Supplementary material: See the Supplementary material for expressions of the multipole moments, near-fields at two metallic layers, and the simulated multipole contributions of four polarizer designs in Figure 5.

The online version of this article offers supplementary material (https://doi.org/10.1515/nanoph-2020-0325). 